Stud. Univ. Babeş-Bolyai Math. 66(2021), No. 3, 441-455

DOI: $10.24193 /$ subbmath.2021.3.03

\title{
New version of generalized Ostrowski-Grüss type inequality
}

\author{
Muhammad Bilal, Nazia Irshad and Asif R. Khan
}

\begin{abstract}
Ostrowski inequality is one of the celebrated inequalities in Mathematics. The main purpose of our study is to generalize the result of Ostrowski-Grüss type inequality for first differentiable mappings and apply it to probability density functions, composite quadrature rules and special means.
\end{abstract}

Mathematics Subject Classification (2010): 26D15, 26D20, 26D99.

Keywords: Ostrowski-Grüss type inequality, Korkine's identity, probability density function.

\section{Introduction}

Literary, such integral inequality that measures the deviation of the integral of the product of two functions and the product of the integrals is referred to Grüss inequality [9].

In 1938, a Ukrainian mathematician A.M. Ostrowski (1893-1986) presented an inequality in his paper [15]. Since then this inequality is known in the history as Ostrowski inequality. A number of authors have written about generalizations of Ostrowski's inequality in the last few years. For example, this topic is considered in $[1,4,5,6,7,11,12,13,16]$. This inequality has been proved to be an exalted and applicable tool for the development of various branches of Mathematics. Integral inequalities that create bounds on the physical quantities are of great importance in the sense that these types of inequalities are not only applicable in integral operator theory, statistics, probability theory, numerical integration, nonlinear analysis, information theory, stochastic analysis and approximation theory but also we can find its applications in different areas of biological sciences, physics and technology.

S.S. Dragomir and S. Wang [7], in the year 1997, gave a proof of the following Ostrowski-Grüss type inequality: 
Theorem 1.1. Let $\phi: I \rightarrow \mathbb{R}$, where $I \subseteq \mathbb{R}$ is an interval, be a mapping differentiable in the interior $I^{\circ}$ of $I$, and let $b_{1}, b_{2} \in I^{\circ}$ with $b_{1}<b_{2}$. If $\alpha \leq \phi^{\prime}(\eta) \leq \lambda, \eta \in\left[b_{1}, b_{2}\right]$ for some constants $\alpha, \lambda \in \mathbb{R}$, then

$$
\begin{aligned}
& \left|\phi(\eta)-\frac{1}{b_{2}-b_{1}} \int_{b_{1}}^{b_{2}} \phi(\xi) d \xi-\frac{\phi\left(b_{2}\right)-\phi\left(b_{1}\right)}{b_{2}-b_{1}}\left(\eta-\frac{b_{1}+b_{2}}{2}\right)\right| \\
\leq & \frac{1}{4}\left(b_{2}-b_{1}\right)(\lambda-\alpha),
\end{aligned}
$$

$\forall \eta \in\left[b_{1}, b_{2}\right]$.

The above inequality gives a relation between the Ostrowski inequality [15] and the Grüss inequality [14].

In the year 2000, by the use of pre-Grüss inequality, N. Ujević, M. Matić and J. E. Pečarić [11] had improved the factor of the right membership of (1.1) with $\frac{1}{4 \sqrt{3}}$ as follows:

Theorem 1.2. Let $\phi: I \rightarrow \mathbb{R}$, where $I$ is an interval such that, $I \subseteq \mathbb{R}$, be a mapping differentiable in the interior $I^{\circ}$ of $I$, and let $b_{1}, b_{2} \in I^{\circ}$ with $b_{1}<b_{2}$. If $\alpha \leq \phi^{\prime}(\eta) \leq$ $\lambda, \eta \in\left[b_{1}, b_{2}\right]$ for some constants $\alpha, \lambda \in \mathbb{R}$, then

$$
\begin{aligned}
& \left|\phi(\eta)-\frac{1}{b_{2}-b_{1}} \int_{b_{1}}^{b_{2}} \phi(\xi) d \xi-\frac{\phi\left(b_{2}\right)-\phi\left(b_{1}\right)}{b_{2}-b_{1}}\left(\eta-\frac{b_{1}+b_{2}}{2}\right)\right| \\
\leq & \frac{1}{4 \sqrt{3}}\left(b_{2}-b_{1}\right)(\lambda-\alpha),
\end{aligned}
$$

$\forall \eta \in\left[b_{1}, b_{2}\right]$.

In the year 2000, by the use of Čbyšev functional, N. S. Barnett et al. [2] improved the result given by N. Ujević, M. Matić and J. E. Pečarić by proving first membership of the right side of (2.1) in terms of Euclidean norm as follows:

Theorem 1.3. Let $\phi:\left[b_{1}, b_{2}\right] \rightarrow \mathbb{R}$ be an absolutely continuous function whose first derivative $\phi^{\prime} \in L_{2}\left[b_{1}, b_{2}\right]$. Then we have the following inequality

$$
\begin{aligned}
& \left|\phi(\eta)-\frac{1}{b_{2}-b_{1}} \int_{b_{1}}^{b_{2}} \phi(\xi) d \xi-\frac{\phi\left(b_{2}\right)-\phi\left(b_{1}\right)}{b_{2}-b_{1}}\left(\eta-\frac{b_{1}+b_{2}}{2}\right)\right| \\
\leq & \frac{\left(b_{2}-b_{1}\right)}{2 \sqrt{3}}\left[\frac{1}{b_{2}-b_{1}}\left\|\phi^{\prime}\right\|_{2}^{2}-\left(\frac{\phi\left(b_{2}\right)-\phi\left(b_{1}\right)}{b_{2}-b_{1}}\right)^{2}\right]^{\frac{1}{2}} \\
\leq & \frac{1}{4 \sqrt{3}}\left(b_{2}-b_{1}\right)(\lambda-\alpha),
\end{aligned}
$$

if $\alpha \leq \phi^{\prime}(\xi) \leq \lambda$ a.e for $\xi$ on $\left[b_{1}, b_{2}\right] \forall \eta \in\left[b_{1}, b_{2}\right]$.

In [2], we can evaluate the pre-Grüss inequality as follows:

$$
T^{2}(\phi, \psi) \leq T(\phi, \phi) T(\psi, \psi),
$$


where $T(\phi, \psi)$ is the Čebyšev functional as defined in [3] and $\phi, \psi \in L_{2}\left[b_{1}, b_{2}\right]$.

In the next section, we provide a generalization of (1.3) and then use it to probability density functions, composite quadrature rules and special means.

\section{Main result}

Theorem 2.1. Let $\phi:\left[b_{1}, b_{2}\right] \rightarrow \mathbb{R}$ be an absolutely continuous function whose first derivative $\phi^{\prime} \in L_{2}\left[b_{1}, b_{2}\right]$, we have

$$
\begin{aligned}
& \mid(1-h)\left[\phi(\eta)-\frac{\phi\left(b_{2}\right)-\phi\left(b_{1}\right)}{b_{2}-b_{1}}\left(\eta-\frac{b_{1}+b_{2}}{2}\right)\right]+h \frac{\phi\left(b_{1}\right)+\phi\left(b_{2}\right)}{2} \\
& +\frac{\phi\left(b_{1}+b_{2}-\eta\right)-\phi(\eta)}{2}+\left(\eta-\frac{b_{1}+b_{2}}{2}\right)\left(\frac{\phi\left(b_{2}\right)-\phi\left(b_{1}\right)}{b_{2}-b_{1}}\right) \\
& -\frac{1}{b_{2}-b_{1}} \int_{b_{1}}^{b_{2}} \phi(\xi) d \xi \mid \\
\leq & {\left[\frac{\left(b_{2}-b_{1}\right)^{2}}{12}\left(3 h^{2}-3 h+1\right)+(1-2 h)\left(\eta-b_{1}\right)\left(\eta-\frac{b_{1}+b_{2}}{2}\right)\right.} \\
& \left.+h(1-h)\left(\eta-\frac{b_{1}+b_{2}}{2}\right)^{2}\right]^{\frac{1}{2}}\left[\frac{1}{b_{2}-b_{1}}\left\|\phi^{\prime}\right\|_{2}^{2}-\left(\frac{\phi\left(b_{2}\right)-\phi\left(b_{1}\right)}{b_{2}-b_{1}}\right)^{2}\right]^{\frac{1}{2}} \\
\leq \quad & \frac{1}{2}(\lambda-\alpha)\left[\frac{\left(b_{2}-b_{1}\right)^{2}}{12}\left(3 h^{2}-3 h+1\right)+(1-2 h)\left(\eta-b_{1}\right)\left(\eta-\frac{b_{1}+b_{2}}{2}\right)\right. \\
& \left.+h(1-h)\left(\eta-\frac{b_{1}+b_{2}}{2}\right)^{2}\right]^{\frac{1}{2}},
\end{aligned}
$$

if $\alpha \leq \phi^{\prime}(\xi) \leq \lambda$ a.e for $\xi$ on $\left[b_{1}, b_{2}\right], \forall \eta \in\left[b_{1}+h \frac{b_{2}-b_{1}}{2}, \frac{b_{1}+b_{2}}{2}\right]$ and $h \in[0,1]$.

Proof. Consider the following kernel defined in $[8] p:\left[b_{1}, b_{2}\right]^{2} \rightarrow \mathbb{R}$

$$
p(\eta, \xi)= \begin{cases}\xi-\left(b_{1}+h \frac{b_{2}-b_{1}}{2}\right), & \text { if } \xi \in\left[b_{1}, \eta\right], \\ \xi-\left(\frac{b_{1}+b_{2}}{2}-h \frac{b_{2}-b_{1}}{2}\right), & \text { if } \xi \in\left(\eta, b_{1}+b_{2}-\eta\right], \\ \xi-\left(b_{2}-h \frac{b_{2}-b_{1}}{2}\right), & \text { if } \xi \in\left(b_{1}+b_{2}-\eta, b_{2}\right] .\end{cases}
$$

By replacing $\phi(\xi)$ with $p(\eta, \xi)$ and $\psi(\xi)$ with $\phi^{\prime}(\xi)$ in Korkine's identity defined as:

$$
T(\phi, \psi):=\frac{1}{2\left(b_{2}-b_{1}\right)^{2}} \int_{b_{1}}^{b_{2}} \int_{b_{1}}^{b_{2}}(\phi(\xi)-\phi(s))(\psi(\xi)-\psi(s)) d \xi d s
$$


we get

$$
\begin{aligned}
& \frac{1}{b_{2}-b_{1}} \int_{b_{1}}^{b_{2}} p(\eta, \xi) \phi^{\prime}(\xi) d \xi-\frac{1}{b_{2}-b_{1}} \int_{b_{1}}^{b_{2}} p(\eta, \xi) d \xi \frac{1}{b_{2}-b_{1}} \int_{b_{1}}^{b_{2}} \phi^{\prime}(\xi) d \xi \\
= & \frac{1}{2\left(b_{2}-b_{1}\right)^{2}} \int_{b_{1}}^{b_{2}} \int_{b_{1}}^{b_{2}}(p(\eta, \xi)-p(\eta, s))\left(\phi^{\prime}(\xi)-\phi^{\prime}(s)\right) d \xi d s .
\end{aligned}
$$

We have,

$$
\begin{aligned}
& \frac{1}{b_{2}-b_{1}} \int_{b_{1}}^{b_{2}} p(\eta, \xi) \phi^{\prime}(\xi) d \xi \\
= & \frac{(1-2 h)}{2} \phi(\eta)+\frac{h}{2}\left[\phi\left(b_{1}\right)+\phi\left(b_{2}\right)\right]-\frac{1}{b_{2}-b_{1}} \int_{b_{1}}^{b_{2}} \phi(\xi) d \xi+\frac{\phi\left(b_{1}+b_{2}-\eta\right)}{2} \\
\frac{1}{b_{2}-b_{1}} \int_{b_{1}}^{b_{2}} p(\eta, \xi) d \xi & =h\left(\frac{b_{1}+b_{2}}{2}-\eta\right)
\end{aligned}
$$

and

$$
\frac{1}{b_{2}-b_{1}} \int_{b_{1}}^{b_{2}} \phi^{\prime}(\xi) d \xi=\frac{\phi\left(b_{2}\right)-\phi\left(b_{1}\right)}{b_{2}-b_{1}}
$$

Identity (2.2) becomes,

$$
\begin{aligned}
& (1-h)\left[\phi(\eta)-\frac{\phi\left(b_{2}\right)-\phi\left(b_{1}\right)}{b_{2}-b_{1}}\left(\eta-\frac{b_{1}+b_{2}}{2}\right)\right]+h \frac{\phi\left(b_{1}\right)+\phi\left(b_{2}\right)}{2} \\
& +\frac{\phi\left(b_{1}+b_{2}-\eta\right)-\phi(\eta)}{2}+\left(\eta-\frac{b_{1}+b_{2}}{2}\right)\left(\frac{\phi\left(b_{2}\right)-\phi\left(b_{1}\right)}{b_{2}-b_{1}}\right) \\
& -\frac{1}{b_{2}-b_{1}} \int_{b_{1}}^{b_{2}} \phi(\xi) d \xi \\
& =\frac{1}{2\left(b_{2}-b_{1}\right)^{2}} \int_{b_{1}}^{b_{1}} \int_{b_{1}}^{b_{2}}(p(\eta, \xi)-p(\eta, s))\left(\phi^{\prime}(\xi)-\phi^{\prime}(s)\right) d \xi d s .
\end{aligned}
$$

$\forall \eta \in\left[b_{1}+h \frac{b_{2}-b_{1}}{2}, \frac{b_{1}+b_{2}}{2}\right]$ and $h \in[0,1]$. 
By using the Cauchy-Schwartz inequality in terms of double integrals, we can write

$$
\begin{aligned}
& \frac{1}{2\left(b_{2}-b_{1}\right)^{2}}\left|\int_{b_{1}}^{b_{2}} \int_{b_{1}}^{b_{2}}(p(\eta, \xi)-p(\eta, s))\left(\phi^{\prime}(\xi)-\phi^{\prime}(s)\right) d \xi d s\right| \\
\leq & \left(\frac{1}{2\left(b_{2}-b_{1}\right)^{2}} \int_{b_{1}}^{b_{2}} \int_{b_{1}}^{b_{2}}(p(\eta, \xi)-p(\eta, s))^{2} d \xi d s\right)^{\frac{1}{2}} \\
& \times\left(\frac{1}{2\left(b_{2}-b_{1}\right)^{2}} \int_{b_{1}}^{b_{1}} \int_{b_{1}}^{b_{2}}\left(\phi^{\prime}(\xi)-\phi^{\prime}(s)\right)^{2} d \xi d s\right)^{\frac{1}{2}} .
\end{aligned}
$$

However,

$$
\begin{aligned}
& \frac{1}{2\left(b_{2}-b_{1}\right)^{2}} \int_{b_{1}}^{b_{2}} \int_{b_{1}}^{b_{2}}(p(\eta, \xi)-p(\eta, s))^{2} d \xi d s \\
= & \frac{1}{b_{2}-b_{1}} \int_{b_{1}}^{b_{2}} p^{2}(\eta, \xi) d \xi-\left(\frac{1}{b_{2}-b_{1}} \int_{b_{1}}^{b_{2}} p(\eta, \xi) d \xi\right)^{2} \\
= & \frac{\left(b_{2}-b_{1}\right)^{2}}{12}\left(3 h^{2}-3 h+1\right)+h(1-h)\left(\eta-\frac{b_{1}+b_{2}}{2}\right)^{2} \\
& +(1-2 h)\left(\eta-b_{1}\right)\left(\eta-\frac{b_{1}+b_{2}}{2}\right)
\end{aligned}
$$

and

$$
\begin{aligned}
& \frac{1}{2\left(b_{2}-b_{1}\right)^{2}} \int_{b_{1}}^{b_{2}} \int_{b_{1}}^{b_{2}}\left(\phi^{\prime}(\xi)-\phi^{\prime}(s)\right)^{2} d \xi d s \\
= & \frac{1}{b_{2}-b_{1}}\left\|\phi^{\prime}\right\|_{2}^{2}-\left(\frac{\phi\left(b_{2}\right)-\phi\left(b_{1}\right)}{b_{2}-b_{1}}\right)^{2} .
\end{aligned}
$$

By using (2.3) - (2.6), we evaluate the first inequality of (2.1).

By using the following Grüss inequality, we proved the second inequality of (2.1)

$$
0 \leq \frac{1}{b_{2}-b_{1}} \int_{b_{1}}^{b_{2}}\left(\phi^{\prime}(\xi)\right)^{2} d \xi-\left(\frac{1}{b_{2}-b_{1}} \int_{b_{1}}^{b_{2}} \phi^{\prime}(\xi) d \xi\right)^{2} \leq \frac{1}{4}(\lambda-\alpha)^{2},
$$

where $\alpha \leq \phi^{\prime}(\xi) \leq \lambda$ a.e for $\xi$ on $\left[b_{1}, b_{2}\right]$.

Remark 2.2. Since

$$
3 h^{2}-3 h+1 \leq 1, \forall h \in[0,1]
$$

and is minimum for $h=\frac{1}{2}$. 
Thus, (2.1) shows an overall improvement in the inequality obtained by Barnett et al. in $[2]$.

We have some remarks of (2.1) in the form of special cases.

Remark 2.3. Under the assumptions of Theorem 2.1 we can get different special cases by putting different values of $h$ and $\eta$.

Special Case 1. For any value of $h$ and $\eta=b_{1}$ or $h=1$ and $\eta=\frac{b_{1}+b_{2}}{2}$ or $h=\frac{1}{2}$ and $\eta=b_{2},(2.1)$ gives trapezoid inequality [17],

$$
\begin{aligned}
& \left|\left(b_{2}-b_{1}\right) \frac{\phi\left(b_{1}\right)+\phi\left(b_{2}\right)}{2}-\int_{b_{1}}^{b_{2}} \phi(\xi) d \xi\right| \\
\leq & \frac{\left(b_{2}-b_{1}\right)^{2}}{2 \sqrt{3}}\left[\frac{1}{b_{2}-b_{1}}\left\|\phi^{\prime}\right\|_{2}^{2}-\left(\frac{\phi\left(b_{2}\right)-\phi\left(b_{1}\right)}{b_{2}-b_{1}}\right)^{2}\right]^{\frac{1}{2}} \\
\leq & \frac{1}{4 \sqrt{3}}(\lambda-\alpha)\left(b_{2}-b_{1}\right)^{2} .
\end{aligned}
$$

Special Case 2. If we take $h=0$ and $\eta=\frac{b_{1}+b_{2}}{2},(2.1)$ becomes mid-point inequality [17],

$$
\begin{aligned}
& \left|\left(b_{2}-b_{1}\right) \phi\left(\frac{b_{1}+b_{2}}{2}\right)-\int_{b_{1}}^{b_{2}} \phi(\xi) d \xi\right| \\
\leq & \frac{\left(b_{2}-b_{1}\right)^{2}}{2 \sqrt{3}}\left[\frac{1}{b_{2}-b_{1}}\left\|\phi^{\prime}\right\|_{2}^{2}-\left(\frac{\phi\left(b_{2}\right)-\phi\left(b_{1}\right)}{b_{2}-b_{1}}\right)^{2}\right]^{\frac{1}{2}} \\
\leq & \frac{1}{4 \sqrt{3}}(\lambda-\alpha)\left(b_{2}-b_{1}\right)^{2} .
\end{aligned}
$$

Special Case 3. If $h=\frac{1}{2}$ and $\eta=\frac{b_{1}+b_{2}}{2},(2.1)$ becomes an averaged mid-point and trapezoid inequality [17],

$$
\begin{aligned}
& \left|\frac{\phi\left(b_{1}\right)+2 \phi\left(\frac{b_{1}+b_{2}}{2}\right)+\phi\left(b_{2}\right)}{4}-\frac{1}{b_{2}-b_{1}} \int_{b_{1}}^{b_{2}} \phi(\xi) d \xi\right| \\
\leq & \frac{\left(b_{2}-b_{1}\right)^{2}}{4 \sqrt{3}}\left[\frac{1}{b_{2}-b_{1}}\left\|\phi^{\prime}\right\|_{2}^{2}-\left(\frac{\phi\left(b_{2}\right)-\phi\left(b_{1}\right)}{b_{2}-b_{1}}\right)^{2}\right]^{\frac{1}{2}} \\
\leq & \frac{1}{8 \sqrt{3}}(\lambda-\alpha)\left(b_{2}-b_{1}\right)^{2} .
\end{aligned}
$$


Special Case 4. If $h=\frac{1}{3}$ and $\eta=\frac{b_{1}+b_{2}}{2},(2.1)$ becomes $\frac{1}{3}$ Simpson's inequality for differentiable function $\phi[17]$,

$$
\begin{aligned}
& \left|\frac{\left(b_{2}-b_{1}\right)}{6}\left[\phi\left(b_{1}\right)+4 \phi\left(\frac{b_{1}+b_{2}}{2}\right)+\phi\left(b_{2}\right)\right]-\int_{b_{1}}^{b_{2}} \phi(t) d t\right| \\
\leq & \frac{\left(b_{2}-b_{1}\right)^{2}}{6}\left[\frac{1}{b_{2}-b_{1}}\left\|\phi^{\prime}\right\|_{2}^{2}-\left(\frac{\phi\left(b_{2}\right)-\phi\left(b_{1}\right)}{b_{2}-b_{1}}\right)^{2}\right]^{\frac{1}{2}} \\
\leq & \frac{1}{12}(\lambda-\alpha)\left(b_{2}-b_{1}\right)^{2} .
\end{aligned}
$$

\section{Applications}

\subsection{For probability density functions}

Let $\mathrm{X}, \phi$ and $\Phi$ be a continuous random variable, the probability density function and the cumulative distribution function, respectively such that $\phi:\left[b_{1}, b_{2}\right] \rightarrow \mathbb{R}_{+}$ and $\Phi:\left[b_{1}, b_{2}\right] \rightarrow[0,1]$, defined as,

$$
\Phi(\eta)=\int_{b_{1}}^{\eta} \phi(\xi) d \xi, \eta \in\left[b_{1}+h \frac{b_{2}-b_{1}}{2}, \frac{b_{1}+b_{2}}{2}\right] \subset\left[b_{1}, b_{2}\right],
$$

and the expectation of the random variable $\mathrm{X}$ on $\left[b_{1}, b_{2}\right]$ is defined as,

$$
E(X)=\int_{b_{1}}^{b_{2}} \xi \phi(\xi) d \xi
$$

Then, we have:

Theorem 3.1. By using above assumptions and if the probability density function $\phi \in$ $L_{2}\left[b_{1}, b_{2}\right]$, we have

$$
\begin{aligned}
& \mid(1-h)\left[\Phi(\eta)-\frac{1}{b_{2}-b_{1}}\left(\eta-\frac{b_{1}+b_{2}}{2}\right)\right]+\frac{h}{2}-\frac{b_{2}-E(X)}{b_{2}-b_{1}} \\
& +\frac{\Phi\left(b_{1}+b_{2}-\eta\right)-\Phi(\eta)}{2}+\frac{1}{b_{2}-b_{1}}\left(\eta-\frac{b_{1}+b_{2}}{2}\right) \mid \\
\leq & \frac{1}{b_{2}-b_{1}}\left[\frac{\left(b_{2}-b_{1}\right)^{2}}{12}\left(3 h^{2}-3 h+1\right)+h(1-h)\left(\eta-\frac{b_{1}+b_{2}}{2}\right)^{2}\right. \\
& \left.+(1-2 h)\left(\eta-b_{1}\right)\left(\eta-\frac{b_{1}+b_{2}}{2}\right)\right]^{\frac{1}{2}}\left[\left(b_{2}-b_{1}\right)\|\phi\|_{2}^{2}-1\right]^{\frac{1}{2}},
\end{aligned}
$$




$$
\begin{aligned}
\leq & \frac{(M-m)}{2}\left[\frac{\left(b_{2}-b_{1}\right)^{2}}{12}\left(3 h^{2}-3 h+1\right)+h(1-h)\left(\eta-\frac{b_{1}+b_{2}}{2}\right)^{2}\right. \\
& \left.+(1-2 h)\left(\eta-b_{1}\right)\left(\eta-\frac{b_{1}+b_{2}}{2}\right)\right]^{\frac{1}{2}}
\end{aligned}
$$

where $m \leq \phi \leq M$ a.e on $\left[b_{1}, b_{2}\right], \forall \eta \in\left[b_{1}+h \frac{b_{2}-b_{1}}{2}, \frac{b_{1}+b_{2}}{2}\right]$

Proof. By putting $\phi=\Phi$ in (2.1), we obtain (3.1).

Corollary 3.2. By using the assumptions of Theorem 3.1 we have,

$$
\begin{aligned}
& \left|(1-h) \operatorname{Pr}\left(X \leq \frac{b_{1}+b_{2}}{2}\right)+\frac{h}{2}-\frac{b_{2}-E(X)}{b_{2}-b_{1}}\right| \\
\leq & \frac{1}{2 \sqrt{3}}\left(3 h^{2}-3 h+1\right)^{\frac{1}{2}}\left[\left(b_{2}-b_{1}\right)\|\phi\|_{2}^{2}-1\right]^{\frac{1}{2}} \\
\leq & \frac{1}{4 \sqrt{3}}\left(3 h^{2}-3 h+1\right)^{\frac{1}{2}}\left(b_{2}-b_{1}\right)(M-m) .
\end{aligned}
$$

\subsection{For composite quadrature rules}

To obtain the estimates of composite quadrature rules, we may use (2.1),

Theorem 3.3. Let $I_{n}: b_{1}=u_{0}<u_{1}<\cdots<u_{n-1}<u_{n}=b_{2}$ be a partition of the interval $\left[b_{1}, b_{2}\right], \Delta u_{j}=u_{j+1}-u_{j}, h \in[0,1], u_{j}+h \frac{\Delta u_{j}}{2} \leq \xi_{j} \leq \frac{u_{j}+u_{j+1}}{2}$, $j=0, \ldots, n-1$. Then,

$$
\int_{b_{1}}^{b_{2}} \phi(\xi) d \xi=S\left(\phi, I_{n}, \xi, h\right)+R\left(\phi, I_{n}, \xi, h\right) .
$$

where

$$
\begin{aligned}
& S\left(\phi, I_{n}, \xi, h\right)=\sum_{j=0}^{n-1}\left[h\left(\frac{\phi\left(u_{j}\right)+\phi\left(u_{j+1}\right)}{2}\right)+\frac{\phi\left(u_{j}+u_{j+1}-\xi_{j}\right)-\phi\left(\xi_{j}\right)}{2}\right. \\
& +(1-h)\left\{\phi\left(\xi_{j}\right)-\frac{\phi\left(u_{j+1}\right)-\phi\left(u_{j}\right)}{\Delta u_{j}}\left(\xi_{j}-\frac{u_{j}+u_{j+1}}{2}\right)\right\} \\
& \left.+\left(\xi_{j}-\frac{u_{j}+u_{j+1}}{2}\right)\left(\frac{\phi\left(u_{j+1}\right)-\phi\left(u_{j}\right)}{\Delta u_{j}}\right)\right] \Delta u_{j} .
\end{aligned}
$$


and

$$
\begin{aligned}
& \left|R\left(\phi, I_{n}, \xi, h\right)\right| \\
\leq & \sum_{j=0}^{n-1}\left[\frac{\Delta u_{j}^{2}}{12}\left(3 h^{2}-3 h+1\right)+(1-2 h)\left(\xi_{j}-u_{j}\right)\left(\xi_{j}-\frac{u_{j}+u_{j+1}}{2}\right)\right. \\
& \left.+h(1-h)\left(\xi_{j}-\frac{u_{j}+u_{j+1}}{2}\right)^{2}\right]^{\frac{1}{2}}\left[\Delta u_{j}\left\|\phi^{\prime}\right\|_{2}^{2}-\left(\phi\left(u_{j+1}\right)-\phi\left(u_{j}\right)\right)\right]^{\frac{1}{2}} \\
\leq & \frac{1}{2}(\lambda-\alpha) \sum_{j=0}^{n-1} \Delta u_{j}\left[\frac{\Delta u_{j}^{2}}{12}\left(3 h^{2}-3 h+1\right)+h(1-h)\left(\xi_{j}-\frac{u_{j}+u_{j+1}}{2}\right)^{2}\right. \\
& \left.+(1-2 h)\left(\xi_{j}-u_{j}\right)\left(\xi_{j}-\frac{u_{j}+u_{j+1}}{2}\right)\right]^{\frac{1}{2}} \cdot
\end{aligned}
$$

Proof. Applying inequality (2.1) on $\xi_{j} \in\left[u_{j}+h \frac{\Delta u_{j}}{2}, \frac{u_{j}+u_{j+1}}{2}\right]$ and summing over $j$ from 0 to $n-1$ and using triangular inequality we get (3.4).

Special Case 1. If $h=0$ in (3.3) and (3.4), $(j=0, \ldots, n-1)$ we have,

$$
S\left(\phi, I_{n}, \xi, h\right)=\frac{1}{2} \sum_{j=0}^{n-1}\left[\phi\left(\xi_{j}\right)+\phi\left(u_{j}+u_{j+1}-\xi_{j}\right)\right] \Delta u_{j}
$$

and

$$
\begin{aligned}
& \left|R\left(\phi, I_{n}, \xi, h\right)\right| \\
\leq & \sum_{j=0}^{n-1}\left[\frac{\Delta u_{j}^{2}}{12}+\left(\xi_{j}-u_{j}\right)\left(\xi_{j}-\frac{u_{j}+u_{j+1}}{2}\right)\right]^{\frac{1}{2}} \\
& \times\left[\Delta u_{j}\left\|\phi^{\prime}\right\|_{2}^{2}-\left(\phi\left(u_{j+1}\right)-\phi\left(u_{j}\right)\right)^{2}\right]^{\frac{1}{2}} \\
\leq & \frac{1}{2}(\lambda-\alpha) \sum_{j=0}^{n-1} \Delta u_{j}^{2}\left[\frac{\Delta u_{j}^{2}}{12}+\left(\xi_{j}-u_{j}\right)\left(\xi_{j}-\frac{u_{j}+u_{j+1}}{2}\right)\right]^{\frac{1}{2}} .
\end{aligned}
$$

Special Case 2. If $\xi_{j}=\frac{u_{j}+u_{j+1}}{2}$ in $(3.3)$ and $(3.4),(j=0, \ldots, n-1)$, we have a perturbed composite mid point and trapezoidal quadrature rule.

$$
\begin{aligned}
S\left(\phi, I_{n}, h\right)= & \sum_{j=0}^{n-1}\left[(1-h) \phi\left(\frac{u_{j}+u_{j+1}}{2}\right)\right. \\
& \left.+h\left(\frac{\phi\left(u_{j}\right)+\phi\left(u_{j+1}\right)}{2}\right)\right] \Delta u_{j}
\end{aligned}
$$


and

$$
\begin{aligned}
& \left|R\left(\phi, I_{n}, h\right)\right| \\
\leq & \frac{1}{2 \sqrt{3}}\left(3 h^{2}-3 h+1\right)^{\frac{1}{2}} \sum_{j=0}^{n-1} \Delta u_{j}\left[\Delta u_{j}\left\|\phi^{\prime}\right\|_{2}^{2}-\left(\phi\left(u_{j+1}\right)-\phi\left(u_{j}\right)\right)^{2}\right]^{\frac{1}{2}} \\
\leq & \frac{1}{4 \sqrt{3}}(\lambda-\alpha)\left(3 h^{2}-3 h+1\right)^{\frac{1}{2}} \sum_{j=0}^{n-1} \Delta u_{j} .
\end{aligned}
$$

Special Case 3. If $h=0$ in $(3.5)$ and $(3.6),(j=0, \ldots, n-1)$, then we have composite midpoint quadrature rule.

$$
S\left(\phi, I_{n}\right)=\sum_{j=0}^{n-1} \Delta u_{j} \phi\left(\frac{u_{j}+u_{j+1}}{2}\right) .
$$

and

$$
\begin{aligned}
& \left|R\left(\phi, I_{n}\right)\right| \\
\leq & \frac{1}{2 \sqrt{3}} \sum_{j=0}^{n-1} \Delta u_{j}\left[\Delta u_{j}\left\|\phi^{\prime}\right\|_{2}^{2}-\left(\phi\left(u_{j+1}\right)-\phi\left(u_{j}\right)\right)^{2}\right]^{\frac{1}{2}} \\
\leq & \frac{1}{4 \sqrt{3}}(\lambda-\alpha) \sum_{j=0}^{n-1} \Delta u_{j}^{2} .
\end{aligned}
$$

Special Case 4. If $h=\frac{3}{10}$ in $(3.5)$ and $(3.6),(j=0, \ldots, n-1)$, then we have a composite mid point and trapezoidal quadrature rule.

$$
\begin{aligned}
& S\left(\phi, I_{n}\right)=\frac{1}{10} \sum_{j=0}^{n-1}\left[7 \phi\left(\frac{u_{j}+u_{j+1}}{2}\right)\right. \\
&\left.+3\left(\frac{\phi\left(u_{j}\right)+\phi\left(u_{j+1}\right)}{2}\right)\right] \Delta u_{j}
\end{aligned}
$$

and

$$
\begin{aligned}
& \left|R\left(\phi, I_{n}\right)\right| \\
\leq & \frac{\sqrt{37}}{20 \sqrt{3}} \sum_{j=0}^{n-1} \Delta u_{j}\left[\Delta u_{j}\left\|\phi^{\prime}\right\|_{2}^{2}-\left(\phi\left(u_{j+1}\right)-\phi\left(u_{j}\right)\right)^{2}\right]^{\frac{1}{2}} \\
\leq & \frac{\sqrt{37}}{40 \sqrt{3}}(\lambda-\alpha) \sum_{j=0}^{n-1} \Delta u_{j}^{2} .
\end{aligned}
$$

Special Case 5. If $h=1$ in (3.5) and (3.6), for $j=0, \ldots, n-1$, we have a composite trapezoidal rule.

$$
S\left(\phi, I_{n}\right)=\frac{1}{2} \sum_{j=0}^{n-1}\left(\phi\left(u_{j}\right)+\phi\left(u_{j+1}\right)\right) \Delta u_{j}
$$


and

$$
\begin{aligned}
& \left|R\left(\phi, I_{n}\right)\right| \\
\leq & \frac{1}{2 \sqrt{3}} \sum_{j=0}^{n-1} \Delta u_{j}\left[\Delta u_{j}\left\|\phi^{\prime}\right\|_{2}^{2}-\left(\phi\left(u_{j+1}\right)-\phi\left(u_{j}\right)\right)^{2}\right]^{\frac{1}{2}} \\
\leq & \frac{1}{4 \sqrt{3}}(\lambda-\alpha) \sum_{j=0}^{n-1} \Delta u_{j}^{2} .
\end{aligned}
$$

\subsection{For special means}

Throughout this section $A, G, H, L, I$ and $L_{p}$ stands for Arithmetic, Geometric, Harmonic, Logarithmic, Identric and $p$-Logarithmic means, respectively, for definitions we refer the readers to [10].

Example 3.4. Let the function $\phi$ be defined by $\phi(\eta)=\eta^{p}, p \in \mathbb{R} \backslash\{-1,0\}$. Then we have,

$$
\begin{gathered}
\frac{1}{b_{2}-b_{1}} \int_{b_{1}}^{b_{2}} \phi(\xi) d \xi=L_{p}^{p}, \\
\frac{\phi\left(b_{2}\right)-\phi\left(b_{1}\right)}{b_{2}-b_{1}}=p L_{p-1}^{p-1}, \\
\frac{\phi\left(b_{1}\right)+\phi\left(b_{2}\right)}{2}=\frac{b_{1}^{p}+b_{2}^{p}}{2}=A\left(b_{1}^{p}, b_{2}^{p}\right), \\
\frac{1}{b_{2}-b_{1}}\left\|\phi^{\prime}\right\|_{2}^{2}=\frac{1}{b_{2}-b_{1}} \int_{b_{1}}^{b_{2}}\left|\phi^{\prime}(\xi)\right|^{2} d \xi=p^{2} L_{2(p-1)}^{2(p-1)}
\end{gathered}
$$

and

Thus, (2.1) becomes,

$$
\frac{\phi\left(b_{1}+b_{2}-\eta\right)}{2}=\frac{(2 A-\eta)^{p}-\eta^{p}}{2}
$$

$$
\begin{aligned}
& \mid(1-h)\left[\eta^{p}-p L_{p-1}^{p-1}(\eta-A)\right]+h A\left(b_{1}^{p}, b_{2}^{p}\right)-L_{p}^{p}+\frac{(2 A-\eta)^{p}-\eta^{p}}{2} \\
& +p L_{p-1}^{p-1}(\eta-A) \mid \\
\leq & |p|\left[\frac{\left(b_{2}-b_{1}\right)^{2}}{12}\left(3 h^{2}-3 h+1\right)+h(1-h)(\eta-A)^{2}\right. \\
& \left.+(1-2 h)\left(\eta-b_{1}\right)(\eta-A)\right]^{\frac{1}{2}} \times\left[L_{2(p-1)}^{2(p-1)}-L_{p-1}^{2(p-1)}\right]^{\frac{1}{2}} .
\end{aligned}
$$

By taking $\eta=A$ in (3.13), we obtain,

$$
\begin{aligned}
& \left|(1-h) A^{p}+h A\left(b_{1}^{p}, b_{2}^{p}\right)-L_{p}^{p}\right| \\
\leq & |p| \frac{\left(b_{2}-b_{1}\right)}{2 \sqrt{3}}\left(3 h^{2}-3 h+1\right)^{\frac{1}{2}}\left[L_{2(p-1)}^{2(p-1)}-L_{p-1}^{2(p-1)}\right]^{\frac{1}{2}},
\end{aligned}
$$


which is minimum for $h=\frac{1}{2}$.

By taking $h=1$, we obtain,

$$
\left|A\left(b_{1}^{p}, b_{2}^{p}\right)-L_{p}^{p}\right| \leq \frac{\left(b_{2}-b_{1}\right)}{2 \sqrt{3}}|p|\left[L_{2(p-1)}^{2(p-1)}-L_{p-1}^{2(p-1)}\right]^{\frac{1}{2}} .
$$

Example 3.5. Let the function $\phi$ be defined by $\phi(\eta)=\frac{1}{\eta},\left(\eta \in\left[b_{1}+h \frac{b_{2}-b_{1}}{2}, \frac{b_{1}+b_{2}}{2}\right] \subset\right.$ $(0, \infty))$. Then,

$$
\begin{gathered}
\frac{1}{b_{2}-b_{1}} \int_{b_{1}}^{b_{2}} \phi(\xi) d \xi=\frac{1}{L}, \\
\frac{\phi\left(b_{2}\right)-\phi\left(b_{1}\right)}{b_{2}-b_{1}}=-\frac{1}{G^{2}}, \\
\frac{\phi\left(b_{1}\right)+\phi\left(b_{2}\right)}{2}=\frac{A}{G^{2}}, \\
\frac{1}{b_{2}-b_{1}}\left\|\phi^{\prime}\right\|_{2}^{2}=\frac{1}{b_{2}-b_{1}} \int_{b_{1}}^{b_{2}}\left|\phi^{\prime}(\xi)\right|^{2} d \xi=\frac{b_{1}^{2}+b_{1} b_{2}+b_{2}^{2}}{3 G^{6}} \\
\frac{1}{b_{2}-b_{1}} \int_{b_{1}}^{b_{2}}\left|\phi^{\prime}(\xi)\right|^{2} d \xi-\left(\frac{\phi\left(b_{2}\right)-\phi\left(b_{1}\right)}{b_{2}-b_{1}}\right)^{2}=\frac{\left(b_{2}-b_{1}\right)^{2}}{3 G^{6}}
\end{gathered}
$$

and

$$
\frac{\phi\left(b_{1}+b_{2}-\eta\right)}{2}=\frac{\eta-A}{\eta(2 A-\eta)} .
$$

Thus, (2.1) takes the form,

$$
\begin{aligned}
& \left|(1-h)\left[\frac{1}{\eta}+\frac{1}{G^{2}}(\eta-A)\right]+\frac{h A}{G^{2}}-\frac{1}{L}+\frac{\eta-A}{\eta(2 A-\eta)}-\frac{(\eta-A)}{G^{2}}\right| \\
\leq & {\left[\frac{\left(b_{2}-b_{1}\right)^{2}}{12}\left(3 h^{2}-3 h+1\right)+h(1-h)(\eta-A)^{2}\right.} \\
& \left.+(1-2 h)\left(\eta-b_{1}\right)(\eta-A)\right]^{\frac{1}{2}} \times \frac{\left(b_{2}-b_{1}\right)}{\sqrt{3} G^{3}} .
\end{aligned}
$$

Put $\eta=A$ in (3.14), we obtain,

$$
\left|(1-h) \frac{1}{A}+\frac{h A}{G^{2}}-\frac{1}{L}\right| \leq \frac{\left(b_{2}-b_{1}\right)^{2}}{6 G^{3}}\left(3 h^{2}-3 h+1\right)^{\frac{1}{2}} .
$$


By taking $\eta=L$ in (3.14), we obtain,

$$
\begin{aligned}
& \left|\frac{2 h A}{G^{2}}-\frac{h L}{G^{2}}-\frac{h}{L}+\frac{L-A}{L(2 A-L)}\right| \\
\leq & {\left[\frac{\left(b_{2}-b_{1}\right)^{2}}{12}\left(3 h^{2}-3 h+1\right)+h(1-h)(L-A)^{2}\right.} \\
& \left.+(1-2 h)\left(L-b_{1}\right)(L-A)\right]^{\frac{1}{2}} \times \frac{\left(b_{2}-b_{1}\right)}{\sqrt{3} G^{3}} .
\end{aligned}
$$

Example 3.6. Let the function $\phi$ be defined by $\phi(\eta)=\ln \eta,\left(\eta \in\left[b_{1}+h \frac{b_{2}-b_{1}}{2}, \frac{b_{1}+b_{2}}{2}\right] \subset\right.$ $(0, \infty))$. Then

$$
\begin{gathered}
\frac{1}{b_{2}-b_{1}} \int_{b_{1}}^{b_{2}} \phi(\xi) d \xi=\ln I, \\
\frac{\phi\left(b_{2}\right)-\phi\left(b_{1}\right)}{b_{2}-b_{1}}=\frac{1}{L}, \\
\frac{\phi\left(b_{1}\right)+\phi\left(b_{2}\right)}{2}=\ln G, \\
\frac{1}{b_{2}-b_{1}} \int_{b_{1}}^{b_{2}}\left|\phi^{\prime}(\xi)\right|^{2} d \xi=\frac{1}{G^{2}}, \\
\frac{\phi\left(b_{1}+b_{2}-\eta\right)-\phi(\eta)}{2}=\ln \left(\frac{2 A-\eta}{\eta}\right)^{\frac{1}{2}}
\end{gathered}
$$

and

$$
\frac{1}{b_{2}-b_{1}} \int_{b_{1}}^{b_{2}}\left|\phi^{\prime}(\xi)\right|^{2} d \xi-\left(\frac{\phi\left(b_{2}\right)-\phi\left(b_{1}\right)}{b_{2}-b_{1}}\right)^{2}=\frac{L^{2}-G^{2}}{L^{2} G^{2}} .
$$

Therefore, (2.1) becomes,

$$
\begin{aligned}
& \left|\ln \frac{\eta^{(1-h)} G^{h}}{I}+h \frac{(\eta-A)}{L}+\ln \left(\frac{2 A-\eta}{\eta}\right)^{\frac{1}{2}}\right| \\
\leq & {\left[\frac{\left(b_{2}-b_{1}\right)^{2}}{12}\left(3 h^{2}-3 h+1\right)+h(1-h)(\eta-A)^{2}\right.} \\
& \left.+(1-2 h)\left(\eta-b_{1}\right)(\eta-A)\right]^{\frac{1}{2}} \times \frac{\left[L^{2}-G^{2}\right]^{\frac{1}{2}}}{L G} .
\end{aligned}
$$


Choose $\eta=A$ in (3.15), we obtain,

$$
\left|\ln \frac{A^{(1-h)} G^{h}}{I}\right| \leq \frac{\left(b_{2}-b_{1}\right)}{2 \sqrt{3}}\left(3 h^{2}-3 h+1\right)^{\frac{1}{2}} \frac{\left[L^{2}-G^{2}\right]^{\frac{1}{2}}}{L G} .
$$

At $h=1,(3.15)$ becomes,

$$
\left|\ln \frac{G}{I}\right| \leq \frac{\left(b_{2}-b_{1}\right)\left(L^{2}-G^{2}\right)^{\frac{1}{2}}}{2 \sqrt{3} L G} .
$$

By taking $\eta=I$ in (3.15), we obtain,

$$
\begin{aligned}
& \left|\ln \frac{G^{h}}{I^{h}}+h \frac{(I-A)}{L}+\ln \left(\frac{2 A-I}{I}\right)^{\frac{1}{2}}\right| \\
\leq & {\left[\frac{\left(b_{2}-b_{1}\right)^{2}}{12}\left(3 h^{2}-3 h+1\right)+h(1-h)(I-A)^{2}\right.} \\
& \left.+(1-2 h)\left(I-b_{1}\right)(I-A)\right]^{\frac{1}{2}} \times \frac{\left[L^{2}-G^{2}\right]^{\frac{1}{2}}}{L G} .
\end{aligned}
$$

Acknowledgment. The research of the first and the third authors is supported by the Higher Education Commission of Pakistan under Indigenous Ph.D. Fellowship for 5000 Scholars, HEC, (Phase-II) (PIN: 518-108309-2PS5-088).

\section{References}

[1] Anastassiou, G.A., Multivariate Ostrowski type inequalities, Acta Math. Hungar., 76(1997), 267-278.

[2] Barnett, N.S., Dragomir, S.S., Sofo, A., Better bounds for an inequality of Ostrowski type with applications, Demonstratio Math., 34(2001), no. 3, 533-542. Preprint RGMIA Res. Rep. Coll., 3(2000), no. 1, Article 11.

[3] Cebyšev, P.L., Sur les expressions approximatives des intégrales définies par les autres prises entre les mêmes limites, Proc. Math. Soc. Charkov, 2(1882), 93-98.

[4] Cerone, P., Dragomir, S.S., Roumeliotis, J., A new generalization of Ostrowski integral inequality for mappings whose derivatives are bounded and applications in numerical integration and for special means, Appl. Math. Lett., 13(2000), no. 1, 19-25.

[5] Cheng, X.L., Improvement of some Ostrowski-Grüss type inequalities, Comput. Math. Appl., 42(2001), no. 1/2, 109-114.

[6] Dragomir, S.S., Sofo, A., An integral inequality for twice differentiable mappings and applications, Tamkang J. Math., 31(2000), no. 4, 257-266. Preprint RGMIA Res. Rep. Coll., 2(1999), no. 2.

[7] Dragomir, S.S., Wang, S., An inequality of Ostrowski-Grüss type and its applications to the estimation of error bounds for some special means and for some numerical quadrature rules, Comput. Math. Appl., 33(1997), no. 11, 16-20.

[8] Ghazi, W., Qayyum, A., A note on new Ostrowski type inequalities using a generalized kernel, Bulletin of Mathematical Analysis and Application, 9(2017), 74-91. 
[9] Grüss, G., Über das Maximum des absoluten Betrages von, Math. Z., 39(1935), no. 1, 215-226.

[10] Irshad, N., Khan, A.R., Shaikh, M.A., Generalized Ostrowski type inequality with applications in numerical integration, probability theory and special means, In Dr. Manuel Alberto M. Ferreira (Eds.), Current Topics in Mathematics and Computer Science, Vol. 9 (74-91). B P International, India-United Kingdom, 2021.

[11] Matić, M., Pečarić, J.E., Ujević, N., Improvement and further generalization of some inequalities of Ostrowski-Grüss type, Comput. Math. Appl., 39(2000), no. 3/4, 161-175.

[12] Milovanović, G.V., Pečarić, J.E., On generalization of the inequality of A. Ostrowski and some related applications, Univ. Beograd Publ., Elektrotehn. Fak. Ser. Mat. Fiz. No. 544-576, (1976), 155-158.

[13] Mitrinović, D.S., Pečarić, J.E., Fink, A.M., Inequalities for Functions and their Integrals and Derivatives, Kluwer Academic Publishers, 1991.

[14] Mitrinović, D.S., Pečarić, J.E., Fink, A.M., Classical and New Inequalities in Analysis, Kluwer Academic Publishers, Dordrecht, 1991.

[15] Ostrowski, A., Über die Absolutabweichung einer differentienbaren Funktionen von ihren Integralmittelwert, Comment. Math. Helv., 10(1938), 226-227.

[16] Ujevic, N., New bounds for the first inequality of Ostrowski-Gruss type and applications, Computers and Mathematics with Applications, 46(2003), 421-427.

[17] Zafar, F., Mir, N.A., A generalization of Ostrowski-Grüss type inequality for first differentiable mappings, Tamsui Oxford J. Math. Sci., 26(2010), no. 1, 61-76.

Muhammad Bilal

University of Karachi, Department of Mathematics,

University Road, Karachi-75270, Pakistan

e-mail: mbilalfawad@gmail.com

Nazia Irshad

Dawood University of Engineering and Technology, Department of Mathematics,

M. A. Jinnah Road, Karachi-74800, Pakistan

e-mail: nazia.irshad@duet.edu.pk

Asif R. Khan

University of Karachi, Department of Mathematics,

University Road, Karachi-75270, Pakistan

e-mail: asifrk@uok.edu.pk 